\title{
Composite Inlays and Onlays Structural, Periodontal, and Endodontic Aspects
}

Author: Stefano Bottacchiari

Publisher: Quintessence Publishing

Language: English

ISBN: 978-88-7492-019-8

Edition: $1 / \mathrm{e}$

Publish Year: 2016

Pages: 584, illustrated

Price: $275.00 €$

Dr. Stefano Bottacchiari's aim is to present his experience regarding indirect composite partial restorations based on predictable protocol in the book entitled "Composite Inlays and Onlays: Structural, Periodontal, and Endodontic Aspects". The book covers all the aspects of this topic in six chapters.

Firstly, following the visit after diagnosis, the author analyses the opportunity to resort to indirect composite partial restorations respecting the dental structure, pulp vitality and periodontal structures.

Adhesive systems require adequate knowledge of the adhesion principles, the chemistry and histology of enamel and dentin, their classification, stability and duration, and a careful protocol based on all this. The second chapter covers this topic.

When indirect composite restorations are made what is very important is the buildup, the cavity geometry, the impressions, cementing and finishing, the relationship with the periodontium and occlusion. These aspects are extensively illustrated with clinical cases in the next following chapters.

The last chapter explains very well all the details and aspects of the cracked tooth syndrome.

This book is based on a multidisciplinary approach about the composite partial restorations of indirect adhesive techniques in the posterior sectors with a rigorous and well illustrated protocol.

Everyone interested in improving his indirect composite restorations skills should consider this book as a useful guide which can provide success in daily practice.

DOI: 10.25241/stomaeduj.2017.4(3).bookreview.6

Florin-Eugen

Constantinescu

DDS, PhD Student

Holistic Dental \& Medical Institute of Bucharest-ROPOSTURO, Bucharest,

Romania

e-mail:

dr.florin.constantinescu@gmail.com 